\title{
Germanica
}

\section{La poésie de Bert Papenfuß vingt ans après la chute du Mur - la politisation d'une poétique}

Die Lyrik Bert Papenfuß' zwanzig Jahre nach der Wende - Politisierung einer Poetik

\section{Cécile Millot}

\section{OpenEdition}

\section{Journals}

Édition électronique

URL : http://journals.openedition.org/germanica/612

DOI : $10.4000 /$ germanica. 612

ISSN : 2107-0784

\section{Éditeur}

Université de Lille

\section{Édition imprimée}

Date de publication : 1 juin 2009

Pagination : 65-84

ISBN : 978-2-913857-23-0

ISSN : 0984-2632

Référence électronique

Cécile Millot, «La poésie de Bert Papenfuß vingt ans après la chute du Mur - la politisation d'une poétique », Germanica [En ligne], 44 | 2009, mis en ligne le 01 juin 2011, consulté le 10 décembre 2020. URL : http://journals.openedition.org/germanica/612 ; DOI : https://doi.org/10.4000/germanica.612

Ce document a été généré automatiquement le 10 décembre 2020.

(c) Tous droits réservés 


\title{
La poésie de Bert Papenfuß vingt ans après la chute du Mur - la politisation d'une poétique
}

\author{
Die Lyrik Bert Papenfuß' zwanzig Jahre nach der Wende - Politisierung einer \\ Poetik
}

Cécile Millot

1 Au moment de la chute du Mur, les amateurs de littérature allemande découvraient en RDA une "scène " littéraire créative et originale, qui n'existait que depuis quelques années et qui a survécu, comme la RDA, un an environ ${ }^{1}$. À Berlin, en particulier dans le quartier du Prenzlauer Berg, mais aussi à Leipzig, à Dresde, à Halle, à Chemnitz (à l'époque Karl-Marx-Stadt), de jeunes auteurs, poètes pour la plupart, qui ne trouvent pas leur place dans les institutions culturelles de la République, se regroupent pour donner vie à une culture parallèle. Des lectures sont organisées dans des appartements privés, ou dans le cadre de paroisses, des revues sont éditées avec les moyens du bord ${ }^{2}$ (textes dactylographiés, œuvres graphiques sérigraphiées - ou parfois originales, cahiers cousus à la main ou à la machine à coudre...), en une centaine, parfois seulement quelques dizaines d'exemplaires.

2 Ce n'est pas une culture d'opposition, ce n'est pas une culture politique, la plupart des artistes s'entendent sur le fait que, ni dans leur pratique littéraire, ni dans leur conception de l'art, ils ne partagent la position des deux générations d'auteurs de la RDA qui les ont précédées. La génération de leurs grands-parents (Brecht lui-même, Johannes R. Becher, Anna Seghers...) a contribué coûte que coûte à l'édification d'un modèle politique, auquel elle s'identifiait de façon militante; la génération de leurs parents (Christa Wolf, Heiner Müller, Volker Braun...), animée d'un fort sentiment de responsabilité pour ce modèle, ne l'a critiqué que pour l'améliorer. Pour ces deux générations, le fait que l'écrivain avait un rôle social et une responsabilité politique était indiscutable. Pour les écrivains dont nous parlons ici ${ }^{3}$, cette évidence a volé en éclats. «Placés par le hasard de leur naissance » (hineingeboren, écrit Uwe Kolbe) dans cet État, ils ne s'intéressent (disent-ils) à la RDA ni pour la décrire, ni pour la défendre, 
ni pour la critiquer. Leur pratique de l'art, et leur mode de vie, sont parallèles, clandestins.

3 Curieusement, il y a une place dans les grandes villes de RDA pour ce monde « alternatif » : la vie n'est pas chère et la tentation de la consommation inexistante, on bénéficie facilement d'un certain nombre de prestations sociales, les appartements vides sont nombreux, une certaine frange de la population s'intéresse vivement à cette pratique culturelle vivante et autonome. Même si le régime autoritaire qu'était la RDA ne tolère que très moyennement la constitution d'un espace public qui échappe à la surveillance de l'État : les "scènes alternatives" sont bien entendu surveillées (et infiltrées ${ }^{4}$ ) par la Stasi, certains artistes ont des démêlés, parfois graves, avec la police.

Dans ce contexte, comment décrire (succinctement) les principes de ces artistes, et en particulier de ces écrivains? L'idée centrale est sans doute, et il faut sans doute le répéter, en particulier ici (à l'ouest) et maintenant (vingt ans après), que la RDA ne les intéresse pas, ils sont farouchement attachés à leur indépendance. Leur thématique reste assez centrée sur leur vie personnelle, leurs sentiments, éventuellement les problèmes de la création. Ils s'échappent dans des mondes parallèles (mondes du Nibelungenlied et des sagas nordiques, coulisses moyenâgeuses, éventuellement sciencefiction). S'il y a une attitude d'opposition, elle se situe, pour les poètes, au niveau de la langue : dans un régime autoritaire, la rébellion contre les normes de la grammaire ou celles de la sémantique est aussi une rébellion. Dans un paysage littéraire encore très marqué par les restes du réalisme socialiste, toute divagation est une provocation. Or les poètes du Prenzlauer Berg soumettent la langue à une expérimentation forcenée, à des variations obstinées. Ils n'hésitent pas à pratiquer le nonsense, à mettre sur pied des réformes de l'orthographe délirantes, à élaborer des langues parallèles. Leur désir de nouveauté s'exprime souvent sous la forme de manifestes, éventuellement inspirés de ceux des futuristes russes. Leur production fait preuve d'une réelle inventivité, la (relative) clandestinité dans laquelle ils vivent leur garantissant un espace de liberté créatrice non négligeable.

Le coup de théâtre que représente la chute du Mur (à laquelle personne ne s'attendait, en tous cas pas à cette date ni sous cette forme) représente donc, pour ce monde artistique et littéraire, une remise en cause totale - et non, comme on a pu se l'imaginer de loin, un soulagement devant l'écroulement d'un régime détesté. Les écrivains du Prenzlauer Berg jouissaient d'une grande liberté d'expression, puisqu'ils ne publiaient rien dans des maisons d'édition officielles. La rencontre avec les lois du marché, au début des années 90, n’a pas été ressentie par ces auteurs comme une libération, mais bien comme la perte de conditions de vie et de travail dans lesquelles ils avaient trouvé un équilibre.

6 Dans les années qui suivent, les uns et les autres s'accommodent comme ils peuvent de ce changement radical. La plupart continuent à écrire. Nous avons choisi de suivre, depuis vingt ans, les aléas de la production de Bert Papenfuß ${ }^{5}$, et nous allons essayer de dégager dans quelle mesure l'évolution de cet auteur peut être considérée comme caractéristique.

7 Connu du temps de la RDA sous le nom de Bert Papenfuß-Gorek, puisque, homme émancipé, il portait joint à son nom celui de son épouse, entre temps de nouveau Bert Papenfuß, il écrit beaucoup, et sans interruption (environ un recueil de poèmes par an depuis 1989). Il publie également de nombreux essais, dans les revues qu'il édite luimême ou au comité de rédaction desquelles il participe. 
8 Pour analyser son évolution, nous nous appuierons sur les derniers recueils d'œuvres poétiques qu'il a publiés: SBZ (1998) et le complexe Rumbalotte / Rumbalotte continua (entre 2004 et 2007) ${ }^{6}$. Il est évident que l'étude de textes littéraires aussi récents, en particulier dans la perspective de l'analyse d'une évolution, pose de nombreux problèmes. L'œuvre est, par la force des choses, incomplète, inachevée, et le recul chronologique est insuffisant pour qu'on puisse porter un jugement. Ce qui semble aujourd'hui être l'amorce d'une évolution significative peut avoir disparu dans quelques années. Nous tenterons donc d'être prudente dans nos conclusions.

9 SBZ paraît dix ans après la chute du Mur - c'est chronologiquement une étape importante. Son titre l'enracine dans la problématique de la (ré-)unification. Il implique un regard en arrière sur la RDA (Sowjetische Besatzungs-Zone jusqu'à la fondation de la RDA), un retour historique. Mais le terme a aussi été largement employé postérieurement à cette fondation (au moins jusqu'en 1970, débuts de la Ostpolitik), par les milieux qui ne reconnaissaient pas la légitimité de la RDA, donc il introduit le thème de l'incompréhension entre l'est et l'ouest, voire du mépris de l'ouest pour l'est, qui est renforcé par le sous-titre: Land und Leute semble annoncer de façon provocatrice un écrit ethnologique, à l'usage de citoyens de la RFA (l'ancienne ou la nouvelle) qui ne connaissent pas leurs concitoyens des nouveaux Bundesländer.

10 Avec le complexe Rumbalotte, Bert Papenfuß semble avoir pris un nouveau départ thématique. Le titre s'explique comme suit : on peut déchiffrer (dit-on dans les milieux bien informés), tatoué sur le sexe de certains marins de la Baltique, le mot de "Rumbalotte ", qui ne devient explicite que lorsque le marin retrouve son entrain : on lit alors « Ruhm und Ehre der baltischen Flotte» (« honneur et gloire à la flotte de la Baltique»). Autour de la flotte de la Baltique gravitent, dans les quatre recueils considérés, les figures des Likedeeler et de Claus Störtebeker, et celle d'Eulenspiegel. Les Likedeeler (en bas allemand: "ceux qui partagent équitablement») sont, au quatorzième siècle, une troupe de corsaires au service (alternativement) du royaume de Suède et du royaume de Danemark, son ennemi, ou (alternativement) une troupe de pirates qui pillent les bateaux des riches villes commerçantes de la Hanse. De Claus Störtebeker, l'un de leurs chefs, (son surnom viendrait de "Stürz' den Becher!» («vide ton gobelet!»), la légende a fait une sorte de Robin des Bois des mers, qui vole aux riches pour redistribuer aux pauvres. L'Eulenspiegel auquel se réfère Papenfuß est celui du roman du Belge francophone De Coster ${ }^{7}$, qui défend la liberté du peuple flamand, opprimé par les Espagnols sous Philippe II. Sous ce quadruple signe de la pornographie et de la boisson, et de la redistribution des richesses et de la lutte contre l'envahisseur, Bert Papenfuß, qui n'a jamais craint de choquer et qui a toujours considéré la production poétique (entre autres) comme une branche de la pratique du canular entre amis, est à son affaire - et semble promettre une poésie plus revendicative, plus ancrée dans la politique qu'elle ne l'était jusqu'à présent.

11 C'est en examinant ces textes, en apparence plus roboratifs et jouissifs que discursifs, que nous allons tenter de constater en quoi Bert Papenfuß, depuis 1989, a évolué, et en quoi cette évolution peut être considérée comme caractéristique d'une situation. Il est évident que Bert Papenfuß reste fidèle au principe de l'expérimentation sur la langue, du jeu avec la langue, même si ses méthodes changent. Il apparait également comme fortement enraciné dans une identité de citoyen de l'ex-RDA, vingt ans après la réunification, ce qui est déjà une attitude militante. L'engagement anarchiste qu'il 
revendique depuis la chute du Mur s'exprime dans son œuvre poétique, mais également dans d'autres pratiques artistiques et publiques.

\section{1- La rupture avec la norme linguistique}

12 Au premier abord, Bert Papenfuß semble s'être assagi depuis les années 80 : ses textes de l'époque frappaient immédiatement par la « réforme de l'orthographe » que Sascha Anderson et lui-même avaient introduites, qui rendait leurs textes pratiquement illisibles (sauf à haute voix). Depuis la chute du Mur, ces graphies destinées à désorienter le lecteur ont disparu. Dans Rumbalotte continua, on remarque non seulement la réintroduction des majuscules et de la ponctuation, mais également la disparition du signe typographique «\&», qui remplaçait la conjonction «et » chez Papenfuß et ses compagnons depuis le début de leur production poétique, et auquel il était resté fidèle. Au nombre des signaux immédiatement visibles d'une continuité, on peut noter par contre que Papenfuß, dans la tradition des revues auto éditées, n'abandonne pas le principe du livre illustré.

Ce qui reste, et de façon évidente, c'est la jubilation dans une utilisation non conforme et irrespectueuse de la langue, un fantastique pied de nez aux conventions, dans des textes qui ne craignent pas les longueurs et dont les redites peuvent fatiguer le lecteur, mais qui témoignent d'une vitalité et d'un esprit de provocation restés intacts. Si telle en est la définition, Bert Papenfuß est et reste un vrai poète.

Plus que la cohérence discursive, le principe de construction de son discours poétique est la sonorité. Papenfuß se lance dans des énumérations sans fin, qui sont des passages de poésie sonore :

«Ich war Katharer und Bogumile,

Bogislaw, Boguljub und Kolup, (...)

Geopolit, Selenopolit,

Heliopolit, Kosmopolit,

Zenitist und Hadjuk-Komit,

Utliger und Likedeeler ... $\wedge^{9}$

(Continua ${ }^{10} 2$, p. 3)

Cette liste présente certes des groupes marginaux (plus ou moins imaginaires) en diverses époques et en divers lieux, desquels le je lyrique se réclame, mais il faut également la considérer comme une collection joyeusement chaotique de mots dont les sonorités s'enchaînent.

Les rimes, les rimes intérieures et les allitérations sont systématisées au point d'en devenir stupides. Elles font partie intégrante de la déconstruction, de la destruction de l'ordonnance de la langue :

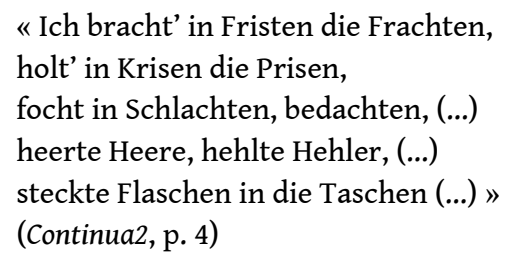

17 Formules symétriques et assonantes, les proverbes absurdes, que Papenfuß a toujours affectionnés, sont encore légion : «was man nicht anpissen kann / geht einen auch nichts an » (SBZ, p. 17). La scatologie (dans cet exemple encore très mesurée - Papenfuß sait faire beaucoup mieux dans ce registre) joue ici toujours son rôle de provocatrice. 

énumérations, les refrains, les variations, qui a toujours intéressé Papenfuß, trouve une nouvelle justification dans le contexte de Rumbalotte. D'une part, ces recueils s'inscrivent expressément dans la tradition des shantys, des chants de matelots destinés à rythmer la manœuvre, où un marin entonne les couplets, le refrain étant repris par l'équipage. D'autre part, les textes de Rumbalotte étaient destinés à donner naissance à un opéra-rock. Le rythme martelé et le triple refrain du poème « Norwegisches Metall » («Métal norvégien »)(Continua 2, p. 13-18), qui autrement apparaîtrait comme assez répétitif, sont légitimés par cet arrière-plan musical.

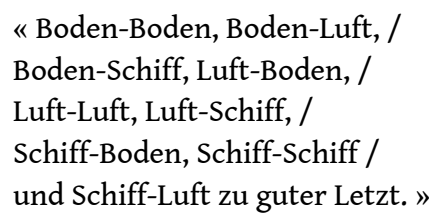
important, en particulier avec leur potentialité de "langue clandestine ». Ce rôle est certainement moins important aujourd'hui, mais langues étrangères et dialectes continuent à fournir à Papenfuß un effet de distanciation efficace. L'usage du Plattdeutsch rend le poème « $7 \times 7=36$ (un titre déjà prometteur) (Continua 2, p. 41 ss.) pratiquement incompréhensible, d'autant plus qu'il est également émaillé de mots arabes et d'autres dont le poète prétend qu'ils sont incas (nous n'avons pas vérifié la véracité de cette affirmation), et de phrases en russe. "Doors of terror » (Rumbalotte, p. 89) est intégralement en anglais, les citations de Sibylla Schwarz, dans «Ist Lieb ein Feur» («Si l'amour est un feu») (Continua 3, p. 22 ss.) sont en allemand baroque, le poème au titre russo-latin « Sub chao КосмичЕСКоГО ШАмАнИзма » (Continua 4, p. 10) commence par cinq lignes de citation en russe, "Ich und wir» ("Je et nous») et "TorTour never stops" (Continua 4, p. 5 et 42) comportent une citation (la même) en japonais... La confusion de la Tour de Babel reste un champ d'expérimentation et un ressort poétique efficace.

De même que de nombreuses langues sont convoquées, certains textes (surtout dans Rumbalotte continua) sont composés presque exclusivement de citations. Nous reviendrons sur leur contenu idéologique (en particulier les textes anarchistes). Mais cette omniprésence du montage, du collage, représente également une forme de victoire de l'absurde sur le sens discursif (aussi bien sur le sens du texte duquel a été extraite la citation que sur celui du texte, volontairement chaotique, auquel elle est intégrée). La saynette (injouable, bien entendu) «Ist Lieb ein Feur» (Continua 3, p. 22 à 32) est composée exclusivement de citations des auteurs suivants, qui sont également les personnages-récitants principaux de la pièce (et les brèves précisions biographiques données par l'auteur ne permettent pas forcément d'établir un rapport entre eux) : Sibylla Schwarz ("poétesse baroque de Greifswald, 1621-1638»), Ernst Fuhrmann («Biosophe, 1886-1956»), Hans Biermann (« Rédacteur de la Ostfriesische Dorfzeitung de Dornum, 1868-?»), Wassili Subbotin (« reporter militaire soviétique, né en $1921 »)$, Helmut Höge («journaliste et musicien ambulant frison de l'est, né en $1947 »)$, Willi Bredel («combattant de la guerre d'Espagne et Président de la Deutsche Akademie der 
Künste, 1901-1964»), et Hans Jaeger ("anarchiste norvégien et artiste bohème, 1854-1910»). La déclamation de ces extraits arbitrairement découpés et montés est interrompue par des indications scéniques absurdes («L'accusatif en particulier est tout autant aboli que, en général, la grammaire de la société. Grondements d'artillerie, bande-son porno“, p. 23), et la source est à chaque fois indiquée en note avec la plus grande exactitude.

$\mathrm{Au}$ détour de Rumbalotte continua 3 se trouvent des citations de récits fantastiques publiés en Union Soviétique dans les années 70 et 80 , de Bert Papenfuß et de quelques autres poètes contemporains, allemands (Peter Wawerzinek), ou autres (norvégiens : Finn Iunker, Tone Avenstroup), de Hegel, du roman de Charles De Coster sur Till Eulenspiegel - etc. On repère dans Rumbalotte continua 2 deux citations de Mao Tsétoung, l'une sur la théorie de la guérilla (p.34), l'autre tirée d'un poème sur la nature (p.39). Au milieu de cette masse de matériau désordonné, le lecteur se perd. De plus, parallèlement (et conséquemment) à l'inflation des citations, le texte étouffe sous les notes : onze, respectivement neuf lignes de texte (en vers courts), pour trente deux, respectivement trente trois lignes de notes (en petits caractères) (Continua 4, p. 4, p. 9). Celles-ci donnent avec une précision exagérée les références éditoriales de chacun des textes auxquels il est fait allusion, plus un certain nombre de détails sur la genèse de ce texte, la biographie de l'auteur cité et autres commentaires de Papenfuß lui-même. La note, sensée par nature éclairer, devient facteur d'obscurité et contribue à donner un caractère chaotique et absurde à un texte qui se refuse à la compréhension immédiate, qui se rebelle contre la dictature du sens.

Papenfuß modernise ses méthodes: la traduction par internet s'avère un excellent moyen pour fabriquer automatiquement de la littérature absurde.

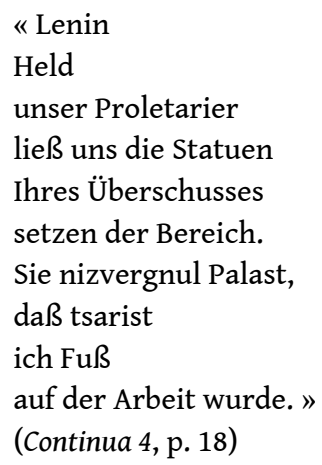

Ce texte a été obtenu en traduisant en anglais, grâce à un programme de traduction (dont Bert Papenfuß donne les références exactes), un poème de propagande russe, puis en retraduisant cette traduction anglaise en allemand. Une traduction plus respectueuse, effectuée par un traducteur de la vieille école (Wolfgang Preuß) donnait

« Lenin, du unser proletarischer Held,

Wir bauen dir ein Denkmal auf dem Platze.

Du hast gestürzt die Zarenwelt

und dich auf die Arbeit gestellt mit dem Fuße.»

(« Lénine, ô notre héros prolétarien, / nous t'érigeons un monument sur la place. /

$\mathrm{Tu}$ as renversé le monde des tsars, / et tu t'es posté sur le travail comme sur un piédestal »).

Le commentaire de l'auteur en note est «Bien entendu c'est une idée stupide de faire des canulars avec des programmes de traduction par internet. Les lecteurs qui se font une certaine idée de la littérature devraient interrompre leur lecture au plus tard ici » 
(Continua 4, p. 18). Toute la question est de savoir quelle idée de la littérature on se fait. Pour Papenfuß, au-delà du canular (forme d'action artistique devant laquelle il ne recule pas), et au-delà, dans cet exemple, de la critique de la rhétorique de propagande, il y a la force subversive de la langue poétique.

Ce dernier exemple nous ramène à la question centrale du rôle idéologique de l'expérimentation sur la langue, du statut de l'avant-garde dans un État autoritaire et dans un État libéral. Dans un État autoritaire (comme l'était la RDA), chaque domaine de la vie est soumis à de fortes contraintes normatives, l'arbitraire du sens est ressenti comme le prolongement d'autres arbitraires, politiques ou sociaux, et la rupture de la norme qu'est l'écriture expérimentale prend une grande valeur. Qu'en est-il dans un État libéral, dans une société permissive, qui, en particulier dans le domaine de la langue, ne se laisse plus provoquer aussi facilement? Certes, les auteurs du Prenzlauer Berg reconnaissent, très tôt après la chute du Mur, qu'ils doivent maintenant partager la pratique du jeu avec la langue avec les agences de publicité - mais Papenfuß luimême affirmait aussi que l'État avait seulement changé de nature, et que si la RDA était caractérisée par l'usage de la «langue de bois du parti » (Parteichinesisch), la RFA l'était par celui de la "langue de bois des banques" (Bankchinesich), et que donc cela ne changeait pas grand-chose ${ }^{12}$. C'est peut-être précisément de cette problématique que les derniers recueils de poésie de Papenfuß portent la marque.

\section{L'affirmation d'une identité de citoyen de l'ex-RDA}

Tout en défendant l'indépendance de sa langue poétique, Bert Papenfuß écrit à partir d'un point de vue qui est de toute évidence celui d'un ancien citoyen de la RDA après la chute de celle-ci, à partir du point de vue d'un Ossi. Il n'hésite pas à faire appel aux souvenirs de son acculturation et de sa socialisation de RDA, il parle des suites économiques et sociales de la réunification en citoyen de l'ex-RDA. Entendons-nous : ses textes ne décrivent pas la RDA, ni la «nouvelle RFA " (Neu-Bundesrepublik, la RFA d'après la réunification), Papenfuß n'est pas un romancier réaliste. Mais l'arrière-plan qui apparaît dans ses textes est bien celui de la RDA et des Nouveaux Bundesländer dans leur spécificité. Et Papenfuß se profile, comme bon nombre de personnes de sa génération, avec une identité (un passé, des racines, une socialisation) différente de celle des citoyens de la "vieille RFA» (Alt-Bundesrepublik, la RFA d'avant la réunification). Le jugement qu'il porte sur la (ré-)unification est par conséquent celui d'un de ces citoyens de la RDA qui ne souhaitaient pas la fusion avec la RFA, et qui critique les conditions dans lesquelles elle a été réalisée, l'abandon délibéré de ce qui aurait pu subsister du potentiel économique, la restitution des biens immobiliers à leurs anciens propriétaires...

C'est le cas plus nettement dans SBZ que dans le complexe de Rumbalotte - mais le sujet n'a pas disparu de la deuxième série de textes, et le recul chronologique manque pour affirmer qu'il y ait une évolution. La nostalgie n'étant guère l'état d'esprit que Papenfuß manifeste, il est difficile de le traiter d'« ostalgique ». Pas plus en 1998 qu'en 2007 (pas plus qu'en 1989), il ne prend la défense de la RDA. Mais ses textes, parfois vengeurs, expriment une identité, et une révolte, dans une perspective militante, politique - ce qui est une nouveauté.

Bert Papenfuß prend plaisir à présenter la RFA et la RDA comme tout aussi négatives, tout aussi mortifères l'une que l'autre, en attribuant sa mort métaphorique à des 
couples d'institutions qui réunissent systématiquement l'est et l'ouest (Rumbalotte p. 28) :

« o mauser, ermordet bin ich worden (...)

(von) volkskammer \& finanzamt

staatssicherheit \& bundestag (...)

revolutionsgarden \& ig-farben $» .$.

30 Le poème «vom sprengen des gartens » («l'arrosage du jardin ») (SBZ, p. 33) élargit la perspective. Il s'agit d'une parodie explicite du poème de Brecht à qui il emprunte son titre, dans lequel le locuteur exprimait le plaisir qu'il prenait à arroser les différentes parties du jardin. Jouant sur l'ambiguïté du terme de sprengen (« arroser» ou «faire sauter »), le locuteur du poème de Papenfuß exprime le plaisir qu'il prend à faire sauter différentes parcelles de terrain, dont l'utilisation par le capitalisme le met en rage :

« (...) grund und boden in die luft zu jagen! Gib mehr als genug \& vergiß nicht die parkplätze \& freiflächen, auch die rückübertragenen nicht, die zugebauten grundstücke! \& übersieh mir nicht zwischen den investitionsruinen die wiedereinrichter, die auch brand haben (...) »

(« (...) faire sauter les terrains et les biens fonciers! Donne plus qu'il n'en faut \& / n'oublie pas les parkings et les espaces non construits, pas / non plus les terrains qui ont été restitués à leur ancien propriétaire, ceux qu'on a / surchargés d'immeubles \& ne laisse pas échapper / entre les immeubles construits pour la spéculation et qui tombent en ruine, les reconstructeurs, / eux aussi, la soif leur met le gosier en feu. »)

31 Ce poème rend bien l'atmosphère politique des années qui ont suivi la chute du Mur. De plus, il engage un dialogue avec trois auteurs qui, eux, se sont toujours présentés comme des poètes engagés - ce qui souligne le nouvel intérêt de Papenfuß pour ce type de question. Le dialogue ne se noue avec Brecht que de façon superficielle, puisque précisément ce poème de Brecht n'est en rien un poème politique - mais le choix de la confrontation avec le père fondateur de la poésie politique de RDA ne peut être fortuit. Un vrai dialogue se noue par contre avec Erich Fried, qui le premier a relevé l'ambiguïté du titre du poème de Brecht ${ }^{13}$. Papenfuß ne fait que mettre en application la métaphore dont Fried avait relevé la possibilité (sprengen dans le sens de «faire sauter »). Et il se trouve de ce fait pour ainsi dire récupéré par Volker Braun qui, lui, s'est toujours considéré et a toujours été considéré comme un poète engagé. En effet, Braun cite ce poème de Papenfuß dans le discours qu'il prononce sur ce dernier à l'occasion de la réception du Prix Erich Fried ${ }^{14}$ par Papenfuß. Braun introduit donc Papenfuß dans la communauté des poètes engagés, avec lui-même et $\mathrm{E}$. Fried, qui ne sont pas des moindres. Nous n'avons pas les moyens de dire si Bert Papenfuß accepte cette réquisition précisément par Volker Braun, qui s'est opposé à lui dans le passé ${ }^{15}$, et dont le discours ne manque pas de maladresse. Mais on ne peut pas nier que, à dix ans de distance, il y ait sinon une parenté, du moins un vrai dialogue, entre " vom sprengen des gartens » de Papenfuß et le très célèbre poème «La propriété » ("Das Eigentum ») de Braun, écrit en 1990 et devenu très rapidement un symbole, qui résume la réaction immédiate des intellectuels de RDA à la chute du Mur. Là où le locuteur du poème de Braun reste tétanisé, comme frappé par le tonnerre devant le changement soudain de répartition de la richesse et son injustice, et devant la perte de l'idéal, celui du poème de Papenfuß, partant de la même analyse (celle du marxiste de RDA), se met à prôner l'action violente.

Sans aller toujours aussi loin dans la question de l'engagement politique, Bert Papenfuß, dans ses poèmes, se souvient de son enracinement, de sa socialisation en 
RDA, revendique son identité d'ancien citoyen de la RDA. Il se moque d'éléments d'idéologie de la RDA, qu'il connaît de l'intérieur. Dans $S B Z$, « La femme de l'est en soi » (" die ostfrau an sich») (p.51) est chantée pendant dix-neuf vers pour son caractère «normal », son indépendance, son sens pratique, son peu de goût pour les grands mots et les scènes, sa capacité de décision, son goût pour le sport... Toutes ces caractéristiques correspondent à l'image que la RDA donnait volontiers de la femme dont le caractère avait été formé par son modèle idéologique et social, que l'indépendance et l'égalité professionnelle et financière devaient nécessairement avoir libérée de tous les problèmes considérés ailleurs comme spécifiquement féminins. En négatif apparaît dans ce poème, comme dans l'idéologie de la RDA, l'image de la femme de RFA, qui, n'étant pas socialement l'égale de l'homme, ne peut que se réfugier dans des remarques capricieuses ou des formules toutes faites prétentieuses, dans la mauvaise humeur - ou dans l'embonpoint. Cet éloge ambigu est fait par un homme (de RDA), qui trouve visiblement bien pratique cette femme qui ne fait pas d'histoires.

Papenfuß se moque de formules de propagande, qu'il connaît par cœur, et qu'il ne reprend que pour s'en distancier : affirmant «Moi et vous, ça ne fait pas un nous ») ("Ich und ihr machen kein Wir») (Continua 4, p. 3), il se distancie de la formule éculée de "la démarche qui mène du «moi » au « nous » ( "der Schritt vom Ich zum Wir »), poncif de la réflexion sur les rapports entre l'individu et le « collectif» (ce point était souvent central dans les discussions sur la littérature, qui devait précisément procurer des modèles d'intégration, voire de soumission). Le poète Papenfuß rappelle ici sans doute de mauvais souvenirs - et les tourne en ridicule en concluant : «Chez moi, vous n'aurez pas de bière » (« Bei mir kriegt ihr kein Bier »).

De même, le simple rappel, sous la forme abrégée sous laquelle les désignaient les élèves, du nom des matières scolaires « PolÖk, DiaMat und HistoMat » (Continua 2, p. 13 à 19) ("politischeökonomie », « dialektischer Materialismus", « historischer Materialismus ») est à la fois un mauvais souvenir et une formule identitaire. L'élève Papenfuß a certainement, comme ceux de ses contemporains qui n'étaient pas parfaitement en phase avec l'idéologie officielle de la RDA, détesté ces matières, puisqu'elles étaient le lieu de l'endoctrinement par excellence. Mais elles ont joué (ou la résistance qu'ils ont tenté de leur opposer a joué) un rôle dans la socialisation du citoyen de RDA qu'est Papenfuß, et leur nom, incompréhensible pour un Wessi, a pour les Ossis une valeur de schibboleth.

Il semble évident que l'intérêt pour la culture russe, héritage de la RDA, dont Papenfuß fait preuve de plus en plus obstinément, alors que les rapports entre l'est de l'Allemagne et la Russie se distendent, est également de sa part une démonstration de son identité d'ex-citoyen de la RDA.

Il y a bien entendu une dimension satirique dans l'utilisation de la littérature de propagande soviétique. Le poème « Choléra des poissons » ("Fisch-xolera ») (Continua 4, p. 18 (note 1)), est consacré à un récit de Boris Lawrenjow, «La dernière balle » (« Der letzte Schuss »), paru en allemand en 1969. Dans ce récit, la garde rouge Marjutka, après un naufrage, vit sur une île une idylle avec un lieutenant des gardes blanches. Mais elle n'hésite pas à sacrifier (au sens propre) son amour, quand elle s'aperçoit que le bateau qui vient les chercher est "blanc», et non pas, comme elle l'espérait, « rouge ». Papenfuß ne prend bien évidemment pas au sérieux ce roman de propagande - c'est lui qu'il soumet à l'épreuve destructrice de la double traduction par internet. Mais il le cite, et, par le choix de ce matériau, par le choix de ce souvenir, il se situe lui- 
même à l'intérieur d'une culture qui relève des démocraties populaires, élément de son identité culturelle qu'il met en avant.

Il cite d'autres récits soviétiques de qualité vraisemblablement modeste, mais qui ont dû former l'horizon culturel des jeunes citoyens de la RDA de son âge : deux recueils de nouvelles fantastiques traduits du russe (parus en RDA en 1979 et en 1984), par exemple (Continua 3, p. 3). Ce rappel est sans doute un souvenir de l'omniprésence du grand frère russe dans tous les domaines, pour une qualité de littérature qui ne justifie pas l'influence qu'on lui a accordée. Mais c'est aussi tout simplement un souvenir des lectures d'enfance qui relie Papenfuß et ses contemporains de RDA, et que ne connaissent pas les hommes de sa génération qui ont grandi en RFA.

Par opposition à la littérature de propagande, donc à une image exagérément positive de l'homme (ou de la femme) soviétique, Papenfuß fait volontiers apparaître dans ses poèmes des situations qui montrent les Russes sous un jour beaucoup moins flatteur. Il reprend là sans aucun doute l'image non officielle, voire interdite, mais obstinée (et on en a beaucoup reparlé depuis la chute du Mur) que les citoyens de la RDA se faisaient des Russes, qui se fondait soit sur leurs souvenirs d'occupation, soit sur leur expérience directe des troupes russes stationnées en RDA (bien que les rapports entre ces troupes et la population locale aient toujours été réduits au minimum) : les Russes sont présentés comme sales, mal élevés et grossiers - mais ils existent dans l'univers de Papenfuß, leur présence à Berlin est régulièrement affirmée.

Dans « kop ab» (" qu'on lui coupe la tête ») (SBZ, p. 28-30), un roi et une reine de Prusse reçoivent un tsar et une tsarine qui savent particulièrement peu se tenir. Avant leur arrivée, la reine cache tout ce qui est fragile, la tsarine porte une robe qu'elle a chipée à une comédienne de Prusse orientale, le tsar est alcoolique et obsédé sexuel, ils partent en emportant le "salon d'ambre ", qu'on ne reverra plus. Après leur départ, le palais de Monbijou est dans un état de destruction digne de deux guerres mondiales. Le refrain $\mathrm{du}$ poème proclame alternativement que «les Russes tapent sur les nerfs des Prussiens » (« die reussen (...) gehen den preussen auf den geist »), ou l'inverse. Il s'agit donc de tout sauf d'un rapport harmonieux - mais le fait que la présence russe à Berlin, le passé historique récent germano-russe et les préjugés des Allemands contre les Russes soient l'objet de ce texte peut être considéré comme une preuve de l'enracinement de Papenfuß dans une culture "de l'est ", qu'il continue à entretenir bien après la réunification.

Dans ce contexte, on ne s'étonnera pas de voir réapparaître deux figures, desquelles Papenfuß s'inspire depuis ses débuts: celle de Quirinus Kuhlmann et celle de Khlebnikov. Quirinus Kuhlmann, poète baroque allemand (1651-1689), cherche à développer une langue qui corresponde à l'originalité de son expérience mystique, donc une langue expérimentale, il intéresse par conséquent déjà beaucoup le Papenfuß de RDA, pour des raisons poétiques (on trouve encore dans Continua 4 le verbe «triumfhosannen", emprunté à Kuhlmann) (p.21, note 6). Mais également, ayant développé une mystique très personnelle, il entreprend de convertir la Russie à cette religion - ce qui ne lui réussit guère (il y est brûlé vif comme hérétique). On voit que Kuhlmann représente pour Papenfuß une figure de passeur, de médiateur entre l'Allemagne et la Russie. Dans les notes du poème qu'il lui consacre ( KuhlmannAltar », «Retable de Kuhlmann », Continua 4, p. 10-12), il cite des passages de Kuhlmann en russe. 
41 La référence à Khlebnikov ${ }^{16}$, elle aussi, est ici à sa place (Continua 3, p. 33-35). Dans une chronologie semi-prophétique loufoque, grâce à un jeu d'additions et de soustractions à partir des millésimes des années où un événement historique s'est produit à Greifswald, Papenfuß, dans l'esprit des «Tables cubo-futuristes du destin » de Khlebnikov, prédit aux bourgeois de la ville un certain nombre de retournements historiques inattendus :

«... à la suite de quoi, en 1964, la section de médecine militaire de la Nationale Volksarmee est intégrée à l'Université Ernst-Moritz-Arndt, ce qui amène en 2377 le triomphe définitif sur la maladie et sur la mort, ce qui d'ailleurs, comme on s'en apercevra, n'arrange pas tout le monde ».

Cet intérêt pour la "face russe " de la culture allemande est un des signes de l'enracinement de Papenfuß dans une culture, donc une identité de RDA. Son intérêt pour la culture et la langue russes (les Wessis sont censés ne s'intéresser qu'aux ÉtatsUnis) est une façon de continuer à vivre son identité de RDA, à la faire vivre et à la développer, même (et surtout) si les objets choisis n'ont rien de commun avec la culture soviétique que la RDA faisait connaître à ses citoyens.

\section{3- L'engagement anarchiste}

Au-delà de l'affirmation d'une identité de RDA, qui est déjà, dans l'Allemagne réunifiée, un acte militant, Papenfuß prend également une position expressément politique.

Pour beaucoup d'anciens citoyens de la RDA, la réunification a des conséquences économiques désastreuses. Les joies de la consommation sont soumises à la nécessité d'un revenu régulier, or le chômage fait des ravages, entreprises et collectivités territoriales sont en faillite, toute une structure sociale s'écroule. Même parmi les plus optimistes ou les plus entreprenants, beaucoup déchantent vite : convertis soudain aux vertus du capitalisme, ils investissent en bourse et perdent leur capital peu de temps après, ou fondent de petites entreprises que, par manque d'expérience, ils ne peuvent sauver de la faillite. Pour de larges couches de la population, quelques années après la chute du Mur, le bilan est négatif - rappelons qu'aujourd'hui encore, le pourcentage de chômeurs est de 6\% dans les anciens Bundesländer et de 12\% dans les nouveaux. Les réponses politiques à cette désillusion vont de l'essor du PDS à celui du NPD.

Un certain nombre d'intellectuels de RDA expriment l'idée que le fait d'avoir vécu sous les deux régimes leur donne, en matière de clairvoyance politique, une avance considérable sur leurs concitoyens qui n'ont connu que la RFA ${ }^{17}$. Et également que le fait d'avoir vécu la chute d'un État de l'intérieur, d'avoir vu s'écrouler du jour au lendemain un système qui semblait inamovible, est une expérience politique irremplaçable.

Pour Bert Papenfuß, la vie dans la «nouvelle RFA » a pour résultat assez immédiat sa conversion à l'anarchisme.

47 S'il y a un texte évidemment et fondamentalement anarchiste de Papenfuß, c'est mors ex nihilo ${ }^{18}$, écrit peu de temps après la chute du Mur, donc peu caractéristique dans l'éventail chronologique de notre étude d'aujourd'hui ${ }^{19}$. Nous nous contenterons de remarquer que, dans les 484 vers de cette œuvre, Papenfuß mène une attaque en règle contre l'argent et contre l'État. Et que c'est sans doute l'un de ses meilleurs poèmes, inventif, drôle, méchant, et efficace. 
Bien que le jeu de mot du titre Rumbalotte continua ancre ce cycle dans la tradition spontanéiste internationale, par référence à la Lotta continua soixante-huitarde italienne, dans $S B Z$ et Rumbalotte Papenfuß semble peiner à trouver un discours qui soit à la fois poétique et anarchiste. Quelques déclarations de principe sont faites hors de tout contexte - leur caractère péremptoire en fait des parodies d'elles-mêmes: «L'anarchiste est à la fin, pas au début de l'évolution de l'humanité » (Continua 2, p. 44), ou «Le communisme est (...) une maladie infantile de l'anarchisme» (SBZ, p. 80), qui retourne la célèbre formule de Lénine.

Quelques fragments d'analyse sont cités, ils se terminent la plupart du temps par une pirouette :

« La politique est finie, elle est discréditée, et à juste titre (« zu Recht »); elle est, en réalité, toujours à droite (« rechts »), il n'y a plus de politique de gauche. (...) La soidisant politique de gauche est un louvoiement opportuniste sur le chemin qui doit mener à une économie de gauche. (...) Le chemin le plus court, en passant par le soulèvement populaire, mène directement à la collectivisation, à la poule au pot et aux petits fours. Les mutins ont suffisamment de canots de sauvetage. » (Continua 3 , p. 18-19).

Les joutes oratoires qui réunissent dans « Ist Lieb ein Feuer » (« Si l'amour est un feu ») (Continua 3, p. 22-32) Ernst Fuhrmann, Willi Bredel et Hans Jäger sont un montage d'une incohérence certes destructrice (donc anarchiste), mais, nous l'avons vu, peu signifiante.

1 Le domaine artistique dans lequel Papenfuß préfère, dans les années 2000, exprimer son engagement, c'est la musique, seule pratique artistique, à ses yeux, véritablement anarchiste.

De ce point de vue, contrairement aux apparences, le cycle « La tête du mercenaire est la queue du chanteur» ("Der Kopf des Söldners ist der Schwanz des Sängers») (Continua 2, p. 19-27) n'est pas un texte sur les démêlés de Papenfuß et de ses musiciens avec les institutions culturelles, et n'est pas un texte sur la musique, mais bien sur les rapports de l'anarchiste avec l'autorité.

53 Le texte le plus clair du cycle porte le titre camouflé (c'est le cas de le dire) de «Comment doit être habillé un musicien rock» («Wie soll ein Rockmusiker angezogen sein ») (Continua 2, p. 24-25). En fait, c'est une profession de foi anarchiste, une déclaration de guerre certes au business culturel, mais à travers lui au monde des affaires en général, au pouvoir politique, et à la mondialisation. Grâce à l'alibi de l'art, le «partisan » qu'est le musicien rock s'introduit jusque « dans la cathédrale » et « dans le palais ». Ses qualités, que le texte énumère, sont :

«1- Irrégularité face aux courants de la mode et aux mass media (...)

2- Mobilité face au business culturel (statique) et au business du divertissement

(flexible) (...)

3- Engagement antipolitique face aux récupérations politiques (...)

4- Caractère local face au nivellement de la mondialisation ».

54 Il est pratiquement un modèle pour la guérilla.

Dans «Les Hell's angels sont-ils des gens comme nous» («Sind Höllenengel Mitmenschen ») (p.19-20), Papenfuß met en parallèle la figure du soldat et celle du musicien rock, tous deux déformés par l'exercice de leur métier (les larmes cèdent la place à un sourire grimaçant) et rendus inhumains par la médiatisation de celui-ci. 

«D'où vient l'armée de métier du divertissement» («Woher kommt das stehende Unterhaltungsheer ») (p. 20-21), un passage extrait d'un dictionnaire d'histoire militaire de RDA est parodié, le contrat qui lie l'organisateur du concert et le groupe musical prenant la place de celui qui lie l'officier et les mercenaires. Le mercenaire, par opposition au partisan, est exploité: il s'est laissé enfermer dans un rapport de dépendance à l'argent, il a perdu sa liberté d'anarchiste. Ce texte est bien entendu également antimilitariste : le soldat de l'avenir sera un « robot à tuer » (p. 22) - mais la conclusion du passage affirme quand même la supériorité de la musique sur l'action armée.

L'un des derniers textes du cycle est d'une clarté argumentative rare chez Papenfuß. I porte sur les récupérations possibles de la musique rock par l'ordre établi ( La musique de variétés peut-elle être explicite ? " « Wie explizit kann Unterhaltungskunst sein? ", Continua 2, p. 23-24). Papenfuß y pose par exemple les principes suivants :

«Toute héroïsation est compatible avec le système. La sublimation des abîmes de l'âme humaine et des convulsions des rapports humains est voulue par la classe dominante du moment - les loisirs doivent être occupés par des problèmes, et la détente doit provenir d'occupations de consommation » (p. 23). souligner : un premier principe est énoncé : «dire simplement ce qui est » (« einfach sagen, wie es ist »). S'il s'avère impossible à appliquer, reste la possibilité suivante :

«Immer schön variieren,

drauflos experimentieren,

nach Ausdruck ringen,

frei von der Leber singen. $»$

(«Se livrer à des variations, / se lancer dans les expérimentations / lutter pour trouver des expressions / chanter de tout son cœur. »)

C'est bien la pratique poétique (et musicale) à laquelle s'attache Papenfuß dans le complexe Rumbalotte.

61 Mais il faut remarquer que sa pratique anarchiste s'exprime également au delà des limites de la poésie et de la musique, dans une activité publique et en particulier éditoriale foisonnante, dont l'un des buts principaux est la propagation de la culture anarchiste. En 1994, il fonde, avec Stefan Döring (qui faisait déjà partie avec lui de la "scène » littéraire du Prenzlauer Berg, du temps de la RDA) la revue Sklaven, qui deviendra Sklaven Aufstand en 1998, puis Gegner en 1999. Puis paraît Zonic, depuis 2004 puis TorTour ${ }^{20}$, en 2005.

62 En particulier avec Sklaven et Gegner, Papenfuß rend un double hommage à Franz Jung : par le choix du titre, et par le fait qu'il édite, dans ces revues, de nombreux extraits de cet auteur. Qui est Franz Jung? Né en 1888, mort en 1963, cet anarchiste dadaïste a eu une vie impressionnante et a laissé une œuvre pléthorique, qui reste peu connue, malgré les efforts de Helmut Heißenbüttel et de Fritz Raddatz en RFA au début des années 60. Homme d'action poussé par ses convictions politiques, il déserte dès 1914, est présent à Berlin lors de la révolution de 1919 aux côté des spartakistes, participe à la fondation du Parti Ouvrier Communiste Allemand (KAPD) en 1920, qui l'envoie négocier en Union Soviétique, rentre en Allemagne, séjourne à Prague, à Vienne, à Genève, à Budapest. Il passera après la Deuxième Guerre mondiale une dizaine d'années aux USA. Journaliste économique engagé, il laisse aussi une œuvre littéraire 
foisonnante : avant 1914, compagnon d'Erich Mühsam, il publie dans Der Sturm et Die Aktion; après 1918, il participe avec Raoul Hausmann aux débuts de la branche berlinoise du mouvement Dada. Tous ces aspects de la personnalité et de la vie de Franz Jung ne peuvent qu'intéresser le poète et l'anarchiste Bert Papenfuss, qui se place en position d'héritier lorsqu'il s'agit de choisir le titre de ses revues. En effet, Franz Jung a formé le projet, en 1927, d'éditer une revue du titre de Sklaven, et a participé à partir de 1924 à l'édition de la revue Der Gegner, qui existait depuis 1919, et avait paru pendant un certain temps au Malik-Verlag, sous la direction de Wieland Herzfelde ${ }^{21}$.

Revendiquant cet héritage anarchiste et dadaïste, la revue Gegner actuelle définit sa ligne éditoriale (ibid.) par des affirmations comme :

«La rébellion contre le régime de la propriété doit s'attaquer à l'esprit malfaisant du néolibéralisme : au réformisme politique, aux institutions littéraires visqueuses, à la jeunesse dorée sur ses planches à voile, à l'intergentillesse infantile et au commercialisme abrutissant. "

Dans presque chacun des numéros de Gegner se trouvent des textes de Franz Jung, de même que de ses contemporains et compagnons de route Raoul Hausmann et Ernst Fuhrmann. Ernst Fuhrmann (1886-1956), lui-même éditeur de quatre revues dont il était le seul auteur (comme Karl Kraus dans Die Fackel), a publié dans les années 30 dans Der Gegner. Touche-à-tout génial, s'intéressant à l'archéologie, à l'ethnologie, à la linguistique, à la botanique, à la géologie, le graphomane Fuhrmann, qui exerça toute sa vie le métier d'éditeur, a écrit soixante-dix livres (des livres scientifiques, de la poésie, de la prose, du théâtre), et laissé des milliers de pages de manuscrits. Bert Papenfuß est co-auteur d'une biographie de Ernst Fuhrmann ${ }^{22}$. Ernst Fuhrmann est l'un des protagonistes (et fournisseur de matériel textuel) de la longue saynette-montage «Ist Lieb ein Feur » (Continua 3, p. 22-32), dont nous avons parlé.

Se consacrant à l'édition des œuvres de Franz Jung ou de Ernst Fuhrmann, Papenfuß contribue à donner ses lettres de noblesse à la culture anarchiste, à la fonder historiquement. Il est également l'un des organisateurs du « Kaffee Burger » qui, depuis 1999, s'est fait un nom comme lieu de théâtre et de musique des quartiers est de Berlin. $\mathrm{Si}$, dans le programme de ce café, on retrouve régulièrement (entre autres) des hommages à Jung et à Fuhrmann, on y trouve également régulièrement des soirées intitulées "Russendisko", qui rappellent l'intérêt de Papenfuß et de ses amis pour la culture russe. Il semble que, en ce début de 2009, Papenfuß envisage de faire ses adieux au Kaffee Burger - on est en droit de s'imaginer qu'il a d'autres projets.

Il pourra s'agir de projets d'action politique ou de projets d'action littéraire - Bert Papenfuß se situe de façon cohérente à la croisée des deux. C'est une position originale par rapport aux poètes de sa génération qui ont partagé son vécu. Parmi ceux de ces auteurs qui sont restés les plus féconds, il est intéressant de constater que les plus connus se sont tournés vers une pratique poétique beaucoup plus classique. Durs Grünbein, par exemple, a atteint aujourd'hui un grand degré de notoriété. Il est légèrement plus jeune que Papenfuß, il commence tout juste à écrire au moment de la chute du Mur - il est sans doute moins marqué, dans sa vie et dans son écriture, par son ancrage en RDA, mais celui-ci est quand même, aujourd'hui encore, nettement identifiable. Et pourtant, la poétique de Grünbein est fondée sur des références classiques, sur les auteurs latins et la mythologie antique, sur la poésie baroque et les sonnets. Uwe Kolbe, qui lui est vraiment de la même génération que Papenfuß et a une biographie comparable (il a fait partie de la «scène alternative » de RDA), même s'il a 
toujours été d'apparence plus discrète, et plus intellectuel, a trouvé sa place depuis la chute du Mur dans le dialogue (éventuellement polémique) avec les grands auteurs allemands, avec Goethe, avec Stefan George, avec les romantiques, dans des problématiques poétologiques, dans la confrontation avec des lieux de mémoires. Ceux des contemporains de Papenfuß qui restent enracinés dans le passé de RDA ont plutôt choisi le terrain de l'autobiographie, comme Gabriele Stötzer ou Andreas Koziol. Bert Papenfuß, maintenant rocker et anarchiste, toujours pilier de bistrot du Prenzlauer Berg, est littérairement et socialement le moins intégré - il n'est pas le poète le moins intéressant.

\section{NOTES}

1. Si les précurseurs se font connaître avant 84, l'apogée doit être située en 88-89.

2. Une liste des principales revues et collectifs d'édition, ainsi que des principaux acteurs culturels de cette époque, se trouve à la fin de l'anthologie de textes tirés de ces revues Vogel oder Käfig sein - Kunst und Literatur aus unabhängigen Zeitschriften in der DDR 1979-1989, herausgegeben von Klaus Michael und Thomas Wohlfarth, Berlin, Druckhaus Galrev, 1992. Sur les revues auto éditées, voir Carola Hähnel-Mesnard, La littérature autoéditée en RDA dans les années 80 - Un espace hétérotopique, Paris, L'Harmattan, 2007.

3. Bert Papenfuß-Gorek, Sascha Anderson, Stefan Döring, Rainer Schedlinski, Jan Faktor, Uwe Kolbe, Gabriele Kachold, Andreas Koziol, Elke Erb, Adolf Endler, Gert Neumann, Kerstin Hensel... Cette liste n'a aucun caractère d'exhaustivité.

4. La découverte du fait que S. Anderson et R. Schedlinski, qui comptaient parmi les membres les plus actifs de la «scène » du Prenzlauer Berg, étaient des agents de la Stasi, jette un moment le discrédit sur les activités de l'ensemble du groupe.

5. Cécile Millot, «La poésie après le «tournant »- un tournant dans la poésie ? L'exemple de Bert Papenfuß et des poètes du Prenzlauer Berg ", dans L'Allemagne unifiée cinq ans après, actes du $28^{\mathrm{e}}$ congrès de l'AGES, édités par Jérôme Vaillant, Presses universitaires de Valenciennes, 1995, p. 373-384.

6. SBZ - Land und Leute, Zeichnungen von Silka Teichert, Berlin, Druckhaus Galrev, 1998; Rumbalotte - Gedichte 1998-2002, Zeichnungen von Ronald Lippock, Basel / Weil am Rhein und Wien, Urs Engeler Editor, 2005; [Rumbalotte continua, 1. Folge, Ostheim / Röhn, Verlag Peter Engstler, 2004 - nous n'avons pas réussi à trouver ce cahier] ; Rumbalotte continua, 2. Folge, Berlin, Karin Kramer Verlag, 2005; Rumbalotte continua, 3. Folge, Verlag Peter Engstler, 2006; Rumbalotte continua, 4. Folge, Karin Kramer Verlag, 2007.

7. Charles De Coster (1827-1879), La Légende et les aventures héroïques, joyeuses et glorieuses d'Ulenspiegel et de Lamme Goedzak au Pays de Flandres et ailleurs, 1867.

8. Cécile Millot, op. cit., p. 377.

9. En particulier dans cette partie consacrée à la déconstruction de la langue, nous nous sommes réservée le droit de ne pas traduire certaines citations.

10. Les références aux recueils de Rumbalotte continua figureront sous l'abréviation "Continua", assortie du numéro du cahier (2, 3 ou 4). Le cahier 3 n'est pas paginé - c'est nous qui avons numéroté les pages, en donnant le numéro 1 à la page de titre. 
11. tendance heavy metal / black metal - nous craignons de sortir ici du domaine de nos compétences.

12. Cécile Millot, op. cit., p. 378-380.

13. "Vom Sprengen », dans le cycle "Beim Lesen der Gesammelten Werke Bertolt Brechts », in Die Beine der größeren Lügen (1969), in Erich Fried, Gesammelte Werke - Gedichte 1, Berlin, Wagenbach, 1993, S. 629.

14. „Auf Papenfuß“, in Volker Braun, Wir befinden uns soweit wohl. Wir sind erst einmal am Ende Äußerungen, Frankfurt am Main, Suhrkamp, 1998, p. 134-138.

15. L'essai de Volker Braun sur Rimbaud contient une critique assez acerbe des poètes du Prenzlauer Berg. "Rimbaud. Ein Psalm der Aktualität», in Volker Braun, Verheerende Folgen mangelnden Anscheins innerbetrieblicher Demokratie, Leipzig, Reclam, 1990, p. 96-121.

16. Sur les rapports entre Papenfuß et Khlebnikov, voir Cécile Millot, "Changement de génération, changement de modèle : la réception de (Maïakovski et de) Khlebnikov par les poètes du Prenzlauer Berg", dans Affinités électives - Les littératures de langue russe et allemande, dir. Kerstin Hausbei et Stefan Gödicke, Paris, Presses de la Sorbonne nouvelle, 2006, p. 211-225.

17. Voir les contributions de Angela Kraus dans L'Allemagne unifiée cinq ans après, actes du $28^{\mathrm{e}}$ congrès de l'AGES, édités par Jérôme Vaillant, Presses universitaires de Valenciennes, 1995 : « La situation à l'est ", p. 385-388, et surtout sa contribution à la table ronde « Où va l'Allemagne unifiée? ", p. 400-401.

18. Bert Papenfuß, mors ex nihilo, Zeichnungen von Jörg Immendorf, Berlin, Druckhaus Galrev, 1994.

19. Voir Cécile Millot, «La poésie après le «tournant » - un tournant dans la poésie ?», op. cit., p. 376.

20. Le Kaffee Burger, quartier général de Bert Papenfuß, est situé Torstraße, anciennement Wilhelm-Pieck-Straße.

21. Voir le site internet de la revue Gegner, onglet « Generallinie ».

22. Ernst Fuhrmann - Der Maschinenwolf - Eine Bio-Bibliographie, herausgegeben von Rembert Baumann, Andreas Hansen, Bert Papenfuß, Rainer Stamm, Berlin, Basis-Druck, à paraître.

\section{RÉSUMÉS}

Bert Papenfuß était dans les années 1980 en RDA l'un des poètes les plus connus de la " scène » littéraire parallèle du Prenzlauer Berg, et il fait partie des auteurs de cette époque qui ont aujourd'hui encore une abondante production littéraire. Contrairement à ses professions de foi apolitiques des années 80, qui s'expliquaient par un refus de la politisation de la littérature attendue par le régime de RDA, Papenfuß écrit depuis la chute du Mur une poésie plus revendicative, plus ancrée dans la politique qu'elle ne l'était jusqu'à présent. Cette évolution est étudiée ici d'après les recueils «SBZ-Land und Leute» (1998) et la série "Rumbalotte " / " Rumbalotte continua»(2004-2007). Ces textes roboratifs et jouissifs montrent en quoi Bert Papenfuß a évolué depuis 1989 et en quoi cette évolution peut être considérée comme caractéristique. Ses méthodes changent, mais le poète reste fidèle au principe de l'expérimentation sur la langue. Il apparaît également, vingt ans après la (ré-)unification, comme fortement enraciné dans une identité de citoyen de l'ex-RDA, ce qui est déjà une attitude 
militante. L'engagement anarchiste qu'il revendique maintenant s'exprime dans son œuvre poétique, mais également dans d'autres pratiques artistiques et publiques.

In den 1980er Jahren war Bert Papenfuß in der DDR einer der bekanntesten Dichter der alternativen literarischen „Szene“ des Prenzlauer Bergs. Er gehört zu den Autoren jener Zeit, die heute noch sehr viel veröffentlichen. Seine apolitischen Glaubensbekenntnisse aus den 80er Jahren waren dadurch zu erklären, dass das DDR-Regime von den Schriftstellern gerade politisches Engagement erwartete - im Gegensatz dazu schreibt Papenfuß seit der Wende eine deutlicher politisch orientierte Lyrik. Diese Entwicklung wird hier an Hand der Bände «SBZ-Land und Leute» (1998) und der Reihe «Rumbalotte» / "Rumbalotte continua » (2004-2007) untersucht. Diese respektlosen und lebenslustigen Texte dokumentieren, dass die von Papenfuß seit 1989 durchgemachte Entwicklung als charakteristisch betrachtet werden kann. Seine Techniken haben sich geändert, aber der Dichter bleibt dem Prinzip der Experimentierung mit der Sprache treu. Zwanzig Jahre nach der (Wieder-)Vereinigung demonstriert er zudem auf geradezu militante Weise seine Verwurzelung in einer DDR-Identität. Das anarchistische Engagement, zu dem er sich heute bekennt, drückt sich in seinem poetischen Werk, aber auch in anderen künstlerischen und öffentlichen Praktiken, aus.

INDEX

Mots-clés : poésie, Prenzlauer Berg

\section{AUTEURS}

CÉCILE MILLOT

Université de Reims - Champagne-Ardenne 